\title{
Prospective head tracking in infants: Head movements, accuracy and timing in relation to a circular object motion
}

Bert JONSSON, Louise RÖNNQVIST and Erik DOMELLÖF

\section{(2) OpenEdition \\ Journals}

Electronic version

URL: http://journals.openedition.org/cpl/4824

DOI: $10.4000 / \mathrm{cpl} .4824$

ISSN: $1379-6100$

Publisher

Centre PsyCLÉ

\section{Electronic reference}

Bert JONSSON, Louise RÖNNQVIST and Erik DOMELLÖF, « Prospective head tracking in infants: Head movements, accuracy and timing in relation to a circular object motion », Current psychology letters

[Online], Vol. 25, Issue 1, 2009 | 2009, Online since 17 March 2009, connection on 08 September 2020. URL : http://journals.openedition.org/cpl/4824 ; DOI : https://doi.org/10.4000/cpl.4824

This text was automatically generated on 8 September 2020

(C) All rights reserved 


\title{
Prospective head tracking in infants: Head movements, accuracy and timing in relation to a circular object motion
}

\author{
Bert JONSSON, Louise RÖNNQVIST and Erik DOMELLÖF
}

This study was supported by a grant from the Swedish Research Council (421-2002-2898) to the second author.

\section{Introduction}

1 One of the most important functions of visual perception is to guide actions towards external objects and to provide information about events that are about to happen (von Hofsten, 1994). Actions like visually tracking or intercepting an object moving through space are cardinal examples of actions that necessitate the implementation of prospective control.

2 There are several studies on young infants' developing ability to track one-dimensional motion (e.g., Jonsson \& von Hofsten, 2003; Shea \& Aslin, 1990; von Hofsten \& Rosander, 1996; 1997; von Hofsten, Kochukhova, \& Rosander, 2007; von Hofsten, Vishton, Spelke, Feng, \& Rosander, 1998). Eye tracking does not reach adult maturity until midadolescence, with a developmental asymmetry in that horizontal smooth pursuit develops earlier than vertical smooth pursuit (Salman, Sharpe, Lillakas, Dennis, \& Steinbach, 2006). However, as early as by two to three months of age, infants show predictive visual eye tracking when following an object moving back and forth in front of them (von Hofsten \& Rosander, 1997). By the age of five to six months, infants are also able to follow an object moving on different linear paths in front of them with predictive head movements, but use the head extensively in comparison to adults (Daniel \& Lee, 1990; Johnson, Amso, \& Slemmer, 2003; Jonsson \& von Hofsten, 2003; von Hofsten \& Rosander, 1997; von Hofsten et al., 1998). 
3 However, almost all everyday tracking involves two dimensions, consisting of both horizontal and vertical components. There is, to our knowledge, only two studies on two-dimensional tracking in infants (Gredebäck, von Hofsten, \& Boudreau, 2002; Gredebäck, von Hofsten, Karlsson \& Aus, 2005). In both of these studies, infants were presented with a computer-displayed object that moved along a circular trajectory. In the first study (Gredebäck et al., 2002), it was shown that 9-month-old infants' eye tracking was functional, i.e. the infants were able to negotiate the two-dimensional motion. In the second, longitudinal study (Gredebäck et al., 2005), eye tracking in 6- to 12 moth old infants was found to be functional through catch-up saccades and thus could be extended to a nonlinear motion. However, in both studies it was also evident that, at higher frequencies, timing, gain and accuracy deteriorated in the vertical dimension. In addition, in the longitudinal study (Gredebäck et al., 2005) there was an evident developmental trend, especially for timing, where vertical eye tracking was demonstrated to be more difficult for the infants at the age of 6 months.

The present study focused on two-dimensional head tracking as expressed in prospective control during a head-unrestrained visual tracking task. As in the studies by Gredebäck and colleagues, the participants tracked an object that moved along a circular trajectory, although in the present study the object was presented on a large vertical screen (see Figure 1). To put emphasis on the developmental issue, we measured two groups of infants (6- and 12-month-old) and one group of adults. The study had two purposes. The first concerned an investigation of the abilities of infants to negotiate the target trajectory when to a large extent being forced to recruit the head. Given the results from infant one-dimensional eye- and head tracking (e.g., Jonsson \& von Hofsten, 2003; von Hofsten \& Rosander, 1996, 1997; von Hofsten et al., 1998), and two-dimensional eye-tracking (Gredebäck et al., 2002; Gredebäck et al., 2005), infants as young as 6 months should be able to negotiate a two-dimensional trajectory. However, in the present task infants had to overcome the inertial properties related to head tracking. This requires a sufficient degree of muscle power, especially in the bilateral sternocleidomastoid and trapezius muscles where the former serves to rotate the head laterally and the latter to move it up and down in the sagittal plane. Thus, given that prospective and precise head tracking requires a sophisticated level of coordination between these two sets of muscles, less mature head tracking for the younger infants (6-month) in comparison to older infants (12-month) and adults was expected. Less mature head tracking were defined as; (i) more extensive head movements (ii) reduced accuracy, and (iii) reduced timing.

5 Although we did not measure eye movements apart from confirming off-line that infants kept a steady gaze on the object from the video recordings, the second purpose was an attempt to relate head tracking to eye tracking. When tracking an object moving in a two-dimensional trajectory, the head and the eyes are free to move in a coordinated fashion. This coordination per se has raised the question whether the same underlying neural controller drives both the eye- and head motor systems (Lanma Bizzi \& Allum, 1978). Dubrovsky and Cullen (2002) argue that the medial superior temporal area (MST), located within the middle temporal area (MT+), and the frontal eye field (FEF) of the prefrontal cortex are two, although perhaps not mutually exclusive, possible candidates from which such coordination emerges. In the studies by Gredebäck and co-workers, it was found that, depending on age and speed of the observed object, gaze deteriorated in the vertical dimension. This occurred for the 
infants but not for the adult control group in the first study (Gredebäck et al., 2002), and increasingly with age for the infants in the second (Gredebäck et al., 2005). This indicates that eye tracking is functional but still developing at 12 months of age. Thus, if a common neural controller drives both the eye- and head motor systems (Dubrovsky \& Cullen, 2002) and has approximately the same course of development, head movements, timing and accuracy should be less stable in vertical head tracking, and an developmental effect should be present in vertical but not in horizontal head tracking.

\section{Methods}

\section{Participants}

6 Fourteen infants and six adults participated in the study. Two infants (one 6-month and one 12-month-old) were excluded from further analysis due to fussiness. The remaining infants were divided into two age groups, one consisting of six infants aged 6 months \pm 1 week ( 2 female and 4 male), and the other of six infants aged 12 months \pm 1 week ( 3 female and 3 male). The adult group consisted of four males and two females with an age range of 26 to 28 years ( $M=27.3$ years). All infants were healthy, fullterm born and with no known medical problems. None of the adults had any known visual or medical problems. All adult participants and all parents of the participating infants gave their informed consent prior to testing. The parents were paid $(60 \mathrm{skr})$ for their participation, the adults participating in the study received no payment.

\section{Apparatus}

7 The object motions were produced by means of an electrically powered motor mounted on the back of a specially designed display board (Figure 1). Driven by the electrical motor, a mechanical arm with a re-adjustable magnet and an attraction magnet on the front of the display board produced the circular motion trajectory of the object. The diameter of the motion was adjustable between 16 to $56 \mathrm{~cm}$. The size of the display board was $72 \times 72 \mathrm{~cm}$, surrounded by a $3 \mathrm{~cm}$ wooden frame and vertically fixed to a stable supporting steel construction.

8 Five differently colored replaceable table tennis balls ( $3.74 \mathrm{~cm}$ in diameter), each with a small bell inside, served as the target. The table tennis ball functioned as a rattle when unattached and became soundless when attached to the magnet on the display board. The object moved at a constant speed of $39 \mathrm{~cm} / \mathrm{s}$ (after an acceleration phase of $0.6 \mathrm{~s}$ ), and with a diameter of $52 \mathrm{~cm}$ for the two infants groups. For the adult group, the diameter of the object motion was altered to $56 \mathrm{~cm}$ so that the visual angle from their eyes would approximately equate that from the infant eyes at the same distance from the moving object. Subsequent to the change of diameter size the velocity increased to a constant speed of $41.6 \mathrm{~cm} / \mathrm{s}$ for the adults (acceleration phase of $0.6 \mathrm{~s}$ ). A revolution was always completed within $4.2 \mathrm{~s}$. The direction of the object was easily changed to move either clockwise or counter-clockwise with a switch on the back of the board. 


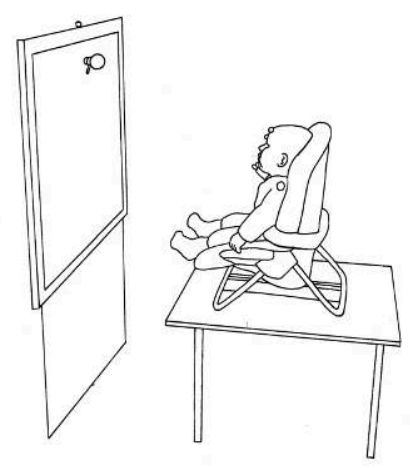

Figure 1. The experimental set-up with an illustration of a 6-month-old infant positioned in front of the display board.

\section{Recording Technique}

9 The movements of interest were recorded by a six-camera, $240 \mathrm{~Hz}$, ProReflex measurement system (Qualisys Inc., Sweden). The cameras were positioned at a distance of approximately 2-3 $\mathrm{m}$ from the participants and monitored infrared reflective markers, with infrared light being emitted by a bank of IREDs located on the cameras. Pressing a hand held button externally triggered the recordings to denote the start of a trial and occurred about $100 \mathrm{~ms}$ before the onset of the object motion. Two markers (diameter $=7 \mathrm{~mm}$ ) were mounted vertically on a piece of hypoallergenic adhesive tape (Micropore), at a distance of $1.5 \mathrm{~cm}$ between each other. The tape was then attached to the participant's forehead, out of view of the participant. Special reflective marker tape was wrapped around the side of the magnet, serving as a marker $($ diameter $=10 \mathrm{~mm})$ for the object. Finally, one reference marker (diameter $=12 \mathrm{~mm})$ was fixed on the top of the display board, in line with the center of the circle.

ll data were sampled at a frequency of $240 \mathrm{~Hz}$, and collected in $7 \mathrm{~s}$ time intervals (one time interval equivalent to an object motion of 1.5 revolutions). For each participant, the total time for testing was about $10 \mathrm{~min}$, with a duration of approximately $15 \mathrm{~s}$ between trials. The two-dimensional (2-D) movement data from each camera were continuously relayed to a Macintosh computer (Power Macintosh 7300/200) for storage. After each measurement session, the system software (MacReflex 3.3) transformed the 2-D data into three-dimensional (3-D) coordinates for further analysis in MATLAB (The Mathworks Inc.).

11 All sessions were video recorded. A video camera (Panasonic FS88) was placed above and behind the display board, zooming in on the participant from a distance of 
approximately $2 \mathrm{~m}$ and focusing on the participant's head and eyes. The purpose of the video recordings was two-folded. Firstly, one monitor was placed behind the display board to enable the experimenter behind the board to see when the participant was looking at the object, and thus to know when to trigger the recordings. Secondly, the video recordings allowed verifications to be made if questions regarding the kinematic recordings arose during the analysis (e.g., if an infant was looking away from the object during a recording interval).

\section{Design and procedure}

12 All adults and infants were presented with 12 trials divided in two counter-balanced blocks. Each block consisted of six trials with the object direction randomized within each block. Thus, each participant was in total exposed to six trials for clockwise object motion and six for counter-clockwise. After the markers had been attached to the participants, they were seated in position for recordings. The 6-month-old infants sat in a standard infant chair (Mothercare) mounted on a table, with the face approximately $43 \mathrm{~cm}$ from the screen and with the eyes in level with the center of the circular motion (Figure 1). The same experimental set-up was created for the 12month-old infants except that they sat in an age-appropriate self-standing baby-chair. The $43 \mathrm{~cm}$ distance between the infant's head and the screen kept the object out of reach. The adults were seated on a pallet, also positioned so that the face was placed at a distance of $43 \mathrm{~cm}$ from the display and the eyes leveled with the center of the circular motion. Throughout the study, classical music provided a soothing background to the sound produced by the apparatus.

Every trial started with the object at the top center of the motion trajectory (Figure 1). The adults were instructed to focus on the object just before it started to move and then to visually track the object in a natural manner. The infants' attention was directed to the object before the ProReflex recording was triggered. If an infant lost interest in the object during the experiment, it was replaced with a differently colored one for the next trial to regain his/her attention. To further maintain the infants' interest, the object was always removed from the display board after each trial and one of the experimenters entertained the infant for the short time period between trials. In the meantime, another experimenter (hidden behind the board) restored the mechanical arm to the starting position and checked that the ProReflex measurement system was ready for the next trial.

\section{Data analysis and statistics}

14 For each trial the on-line video recordings could be synchronized with the kinematic recordings as a green light indicator, simultaneously switched on with the triggering of the ProReflex system, was in constant view of the video camera. This was especially important for the two infant groups as the infants sometimes lost interest and/or looked away from the object during an ongoing trial. Initially, all kinematic data was smoothed by using a second-order $10 \mathrm{~Hz}$ dual pass Butterworth filter. Relevant data were then analyzed by means of customised MATLAB programs. Onset of object motion was always defined as the position in time when the velocity exceeded $20 \mathrm{~mm} / \mathrm{s}$, as was the onset of the head movement. 

head movement for each age group. A consistent pattern of large head movements was taken as an indication of low-level muscular control and interpreted as immature head tracking. The accuracy in head tracking was more explicitly estimated by calculating the time difference between target and center of the head, projected on the presentation plane. This was done at four positions of the circle, corresponding to a clock minute hand position at the times 3, 6, 9 and 12 (se figure 2, a to c). To describe the temporal relationship (timing) between head movement and object motion during a full revolution, time lag between head- and object velocities was calculated separately in both the horizontal and vertical planes. A small time lag indicates a close fit between head movement and target motion. in the analysis. For the analysis of head movements the participants had to track the object for at least half a revolution $\left(180^{\circ}\right)$. The number of trials included in this analysis was 69 for the 6-month-olds, 69 for the 12-month-olds, and 72 for the adults. For accuracy, the participant had to consistently track the object at least from position 1 or 2 to position $4\left(270^{\circ}\right)$. Trials included in this analysis were 47 for the 6-month-olds, 28 for the 12-month-olds, and 72 for the adults. For the analysis of overall timing, an additional restriction was applied through exclusion of trials not including consistent head tracking during a full $360^{\circ}$ cycle in each measured dimension. As a consequence, one additional infant had to be excluded from the 12-month-old infant group. The subsequent analysis included 43 trials for the 6-month-olds, 24 for the 12-month-olds, and 71 for the adults. The initial analysis revealed no effects of direction $(p>.05)$. This factor was subsequently excluded from further analysis and all results are presented collapsed over direction. One-way analyses of variance (ANOVAs) were used to analyze effects of age. If significant age effects were found, Bonferroni adjusted $t$-tests $(\mathrm{p}<$. 0167) were carried out. 

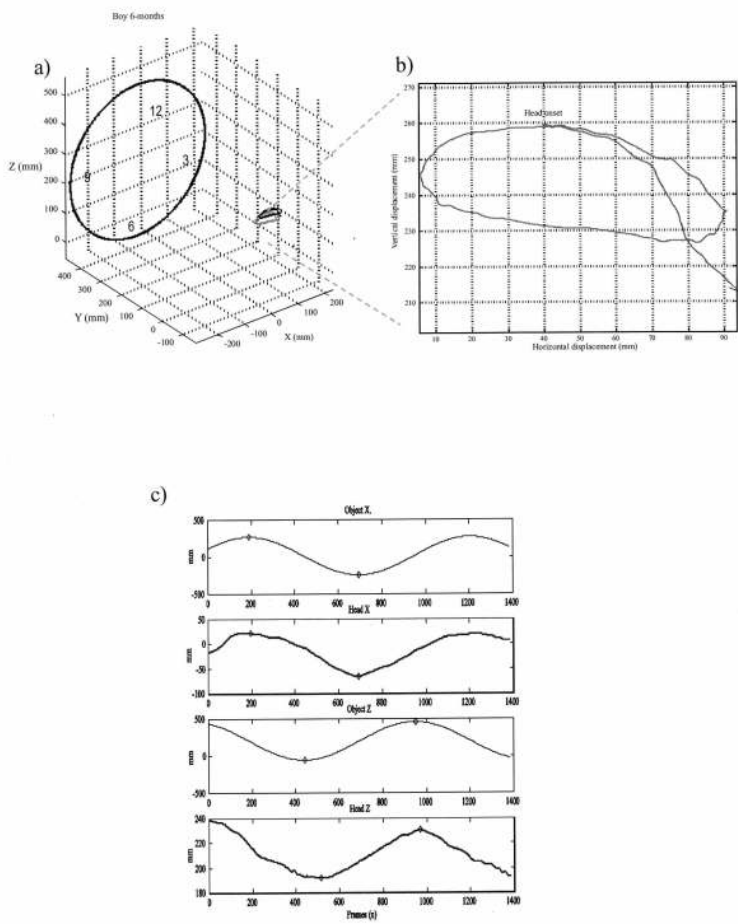

Figure $2(a-c)$

a) 3-D example of a 6-month-old infant's head tracking movement. The circle is the object's trajectory

(moving in a clockwise direction in this example).

b) A zoom in on the head tracking movement (horizontal and vertical) seen in fig. 2 a.

c) The time lag between the object and the head in the four predefined quadrants of the circle. The figure shows head movement and object motion in the horizontal plane at 3 and 9 o'clock (object $X$ and head X) and the vertical plane at 6 and 12 o'clock (object $Z$ and head Z) over time ( 240 frames $=1$ $\mathrm{sec}$ ). The time lags of the head are illustrated by four dots corresponding to four dots on the object sinus shape.

\section{Results}

\section{Head movements}

Figure 3 (a to $\mathrm{c}$ ) shows representative head movements made by two infants from respective age group and one adult. Total cumulative 3-D distance was defined as the dependent variable. The distances covered by the two infant age groups are much greater than that for the adult one, particularly the excursion along the X-axis. A significant age effect for the cumulative head movements was found, $F(2,15)=5.31, p=$ .018. Post-hoc analysis showed that the 6-month-old infants moved their heads a significantly longer distance $(\mathrm{M}=336 \mathrm{~mm})$ in comparison to the adults $(\mathrm{M}=172 \mathrm{~mm})$. However, no significant difference between the 6- and 12-month-old $(\mathrm{M}=293 \mathrm{~mm})$ infants was found. 

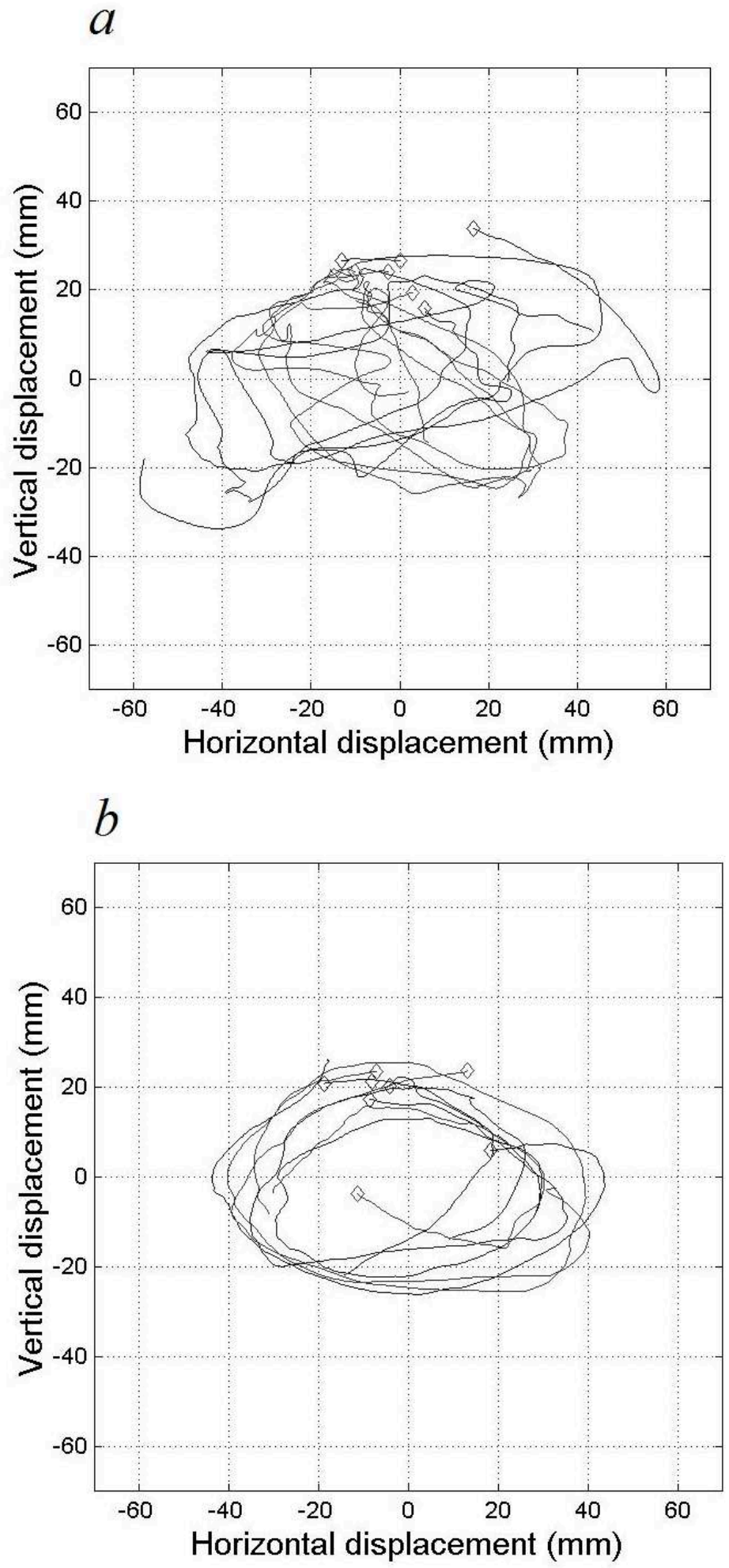


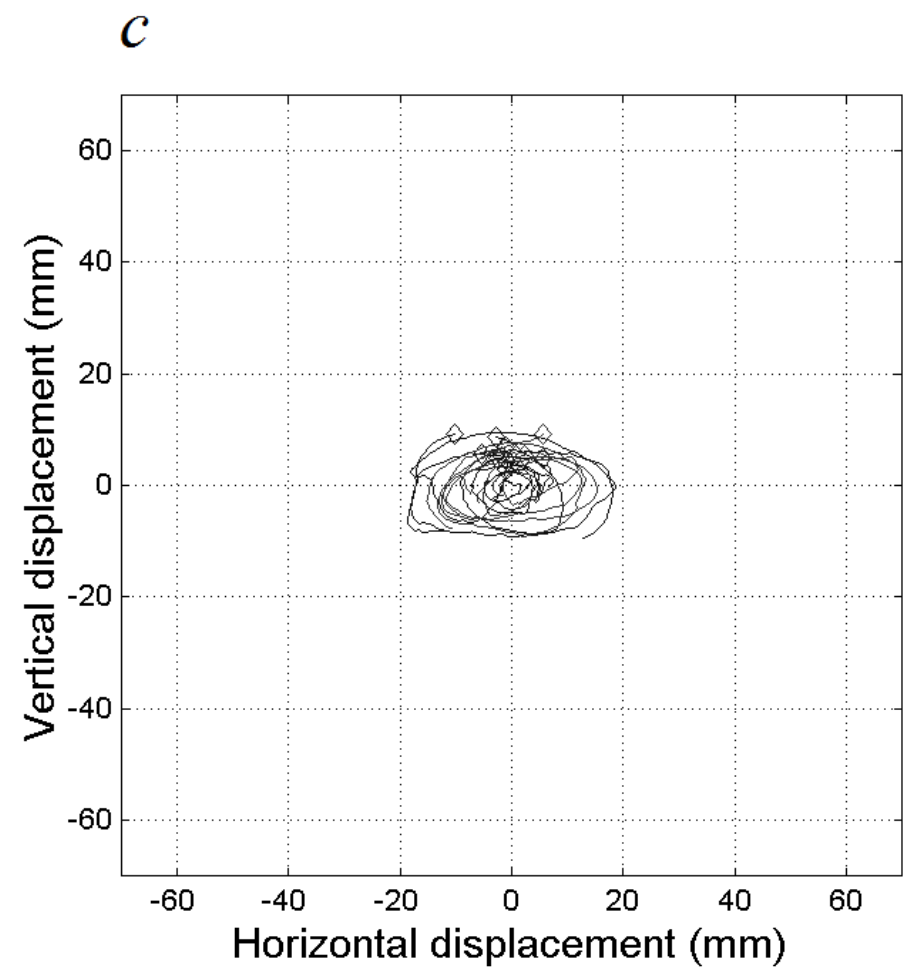

Figure 3 (a-c). Typical examples of head movements made by two infants and one adult, with the movements plotted as co-joint displacements along the $\mathrm{X}$ - and $\mathrm{Y}$-axes. Each diamond-shaped symbol indicates the start of head movements with tracking in both clockwise and counter-clockwise directions.

a) 6-month-old infant (6 revolutions)

b) 12-month-old infant (6 revolutions)

c) Adult (8 revolutions)

\section{Accuracy}

Figure 4 shows the means and standard errors for the time differences between target and center of the head at each of the four positions. This time difference was defined as the dependent variable (i.e., accuracy in terms of temporal deviation of the head while tracking the target motion). Overall, adults had the least lag, followed by the 12-montholds, and with the 6-month-old infants displaying the largest time difference between the head and object. Figure 4 also shows that in the second horizontal position (either 3 or 9 o'clock) the infant groups are almost on target. An ANOVA with positions as within-subject variables and age as between-subject variable revealed effects of age and position on accuracy; Age, $F(2,15)=7.73 p=.005$; Position, $F(3,45)=8.85, p=.001$. No significant interaction effect between age and position was found. Post-hoc analysis of age displayed a significant difference between adults and 6-month-old infants. A contrast analysis with the first position (either 3 or 9 o'clock) as reference category showed that the time difference in the third position (either 3 or 9 o'clock) differed significantly from the first reference position, $F(1,15)=32.21 \mathrm{p}=.0001$. Repeated measurement analysis for each age group revealed an age effect of positions on accuracy for both 6 -month-old, $\mathrm{F}(3,15)=4.16, \mathrm{p}=.025$, and 12-month-old infants, $\mathrm{F}(3,15)=4.14, \mathrm{p}=.025$, respectively. However, no effects were found for the adults. The effect of age shows that both 6-and 12-month-old infants to a larger extent have problems in maintaining accuracy over a full revolution. 


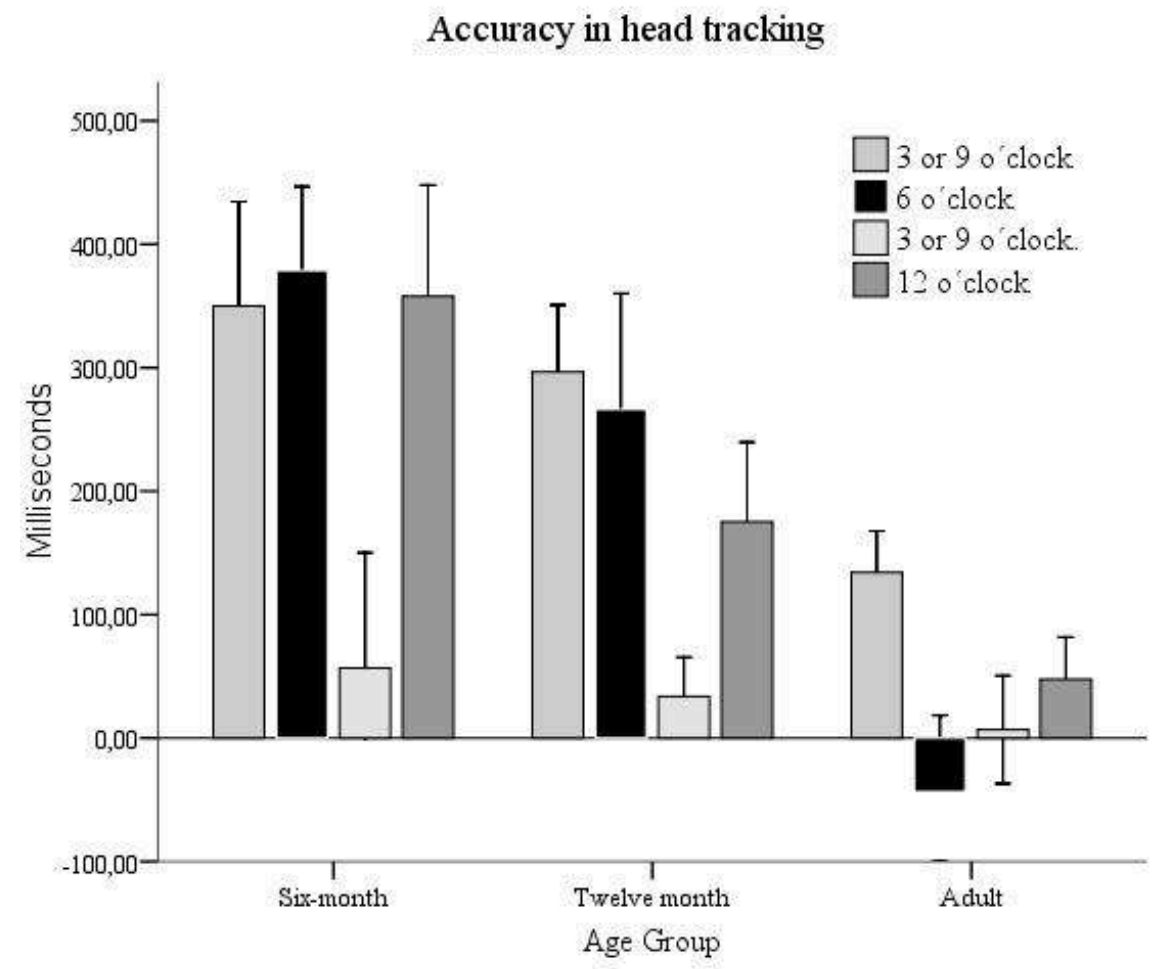

Figure 4. Time differences between target and center of the head, with the differences plotted at each of four predefined positions corresponding to the clock minute hand position at the times 3, 6, 9 and 12. Error bars represent the standard error of the mean.

\section{Timing}

Figure 5 presents the means and standard errors for the time lags between head- and object velocity in the horizontal and vertical planes. In the analyses of timing (i.e., temporal lagging of head velocity in relation to target velocity) time lag constituted the dependent variable, with less time lag indicating a better overall timing between object and head tracking. It is shown that the time lag in the Y-dimension is shortest among the adults, followed by the 12-month-olds, and with the 6-month-olds having the largest time lag. The ANOVAs for the $\mathrm{Y}$-axis also revealed a significant effect of age on time lag, $\mathrm{F}(2,14)=11.34, \mathrm{p}<.001$. However, no age effect was found for the $\mathrm{X}$-axis. Posthoc analyses revealed that the time lag on the $\mathrm{Y}$-axis differed significantly between adults and 6-month-old infants. 
Timing between head tracking and object motion

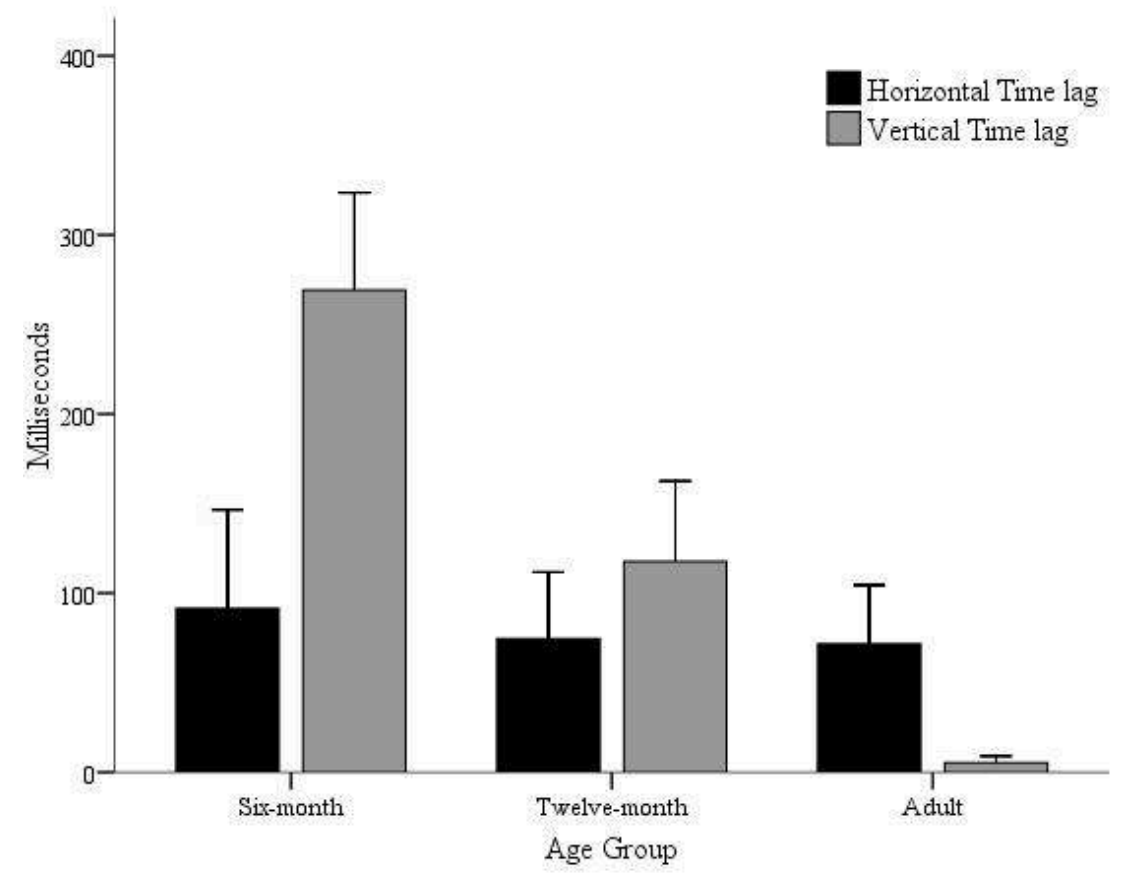

Figure 5 Overall time lags between head- and object velocity in the horizontal and vertical planes. Error bars represent the standard error of the mean.

\section{Discussion}

The results show that prospective head tracking is functional and can be extended to a two-dimensional motion as early as from 6 months of age. However, a developmental trend is also evident. In comparison to adults, young infants; (i) display more extensive head movements, (ii) are less accurate, and (iii) have a less developed timing between head movements and object motion in the vertical dimension. Although our hypothesis that the 6-month-old infants' head tracking would differ significantly from that of the 12-month-olds turned out to be inaccurate in some of the analyses, a pattern characterized by the 6-month-old infants having the least mature head tracking was present for all parameters studied. In addition, a reasonable assumption is that an increased power in the analysis (i.e., more participants in the experiment) may well reveal an age effect between 6- and 12-month-old infants.

The use of extensive head movements in young infants is in line with previous research on one-dimensional horizontal head tracking (Daniel \& Lee, 1990; Jonsson \& von Hofsten, 2003; von Hofsten, Feng, \& Spelke, 2000; von Hofsten \& Rosander, 1997). Jonsson and von Hofsten (2003) showed that 6-month-old infants use the head extensively when tracking a target moving horizontally over the visual field. The head moved almost as much as the observed object did, only moving less at the very extreme initial part of the object trajectory. With regard to the present study, as shown in Figure 3 (a-c), infants displayed more extensive head movements compared to adults. Both infants and adults, infants in particular, covered a greater distance in the 
horizontal than the vertical dimension, indicating less constraints in the execution of horizontal head movements.

The effects of age in the accuracy analyses show that two-dimensional head tracking is not yet fully mature at 6 - and 12 months of age. Given the extensive head movements, one plausible explanation could be that this is due to difficulties in coordinating the two sets of still relatively immature muscles involved, i.e. the bilateral sternocleidomastoid and trapezius muscles. Overcoming the inertia of the head requires a sufficient degree of power in these muscles. The sternocleidomastoid serve to rotate the head laterally and the trapezius to move it up and down in the sagittal plane. Smoothly tracking an object moving in a circular trajectory across a vertical plane would require a sophisticated level of coordination between the two sets of muscles. For example, with the object starting from above and moving in a clockwise direction, tracking it to its lowest point would involve activation of the trapezius muscles. As the observer turns the head towards to the left side, the right sternocleidomastoid activate the head to move from right to left in order to maintain tracking with its left side homologue being subject to reciprocal inhibition. When the object is moving upwards, the trapezius muscles will be the main activators. Beyond midway in the upward trajectory the left sternocleidomastoid will become increasingly active so as to bring the head in alignment with the object's original starting position. Consequently, when the object is moving along a circular trajectory, the precision in head tracking varies depending on position.

However it cannot be ruled out that the effects are reflecting an inability to integrate the two-dimensional features of the circular object motion. If the tracking system is responsive only to the immediately preceding motion of the object (the local characteristic) and tries to negotiate the object by extrapolating far ahead, there is a risk that the extrapolation will be incoherent with the previous trajectory, i.e. the circular object motion (Price \& Gilden, 2000). In line with this reasoning, von Hofsten et al. (1998) found that 6-month-old infants had difficulties in disengaging from a linear extrapolation when the object abruptly turned, despite several trials. Thus, disengaging from a linear extrapolation when the object, without cues, abruptly turns requires a global understanding of the whole trajectory and not just a prediction based on the local characteristics of the moving object.

Manipulating the head involvement by varying the object motion diameter and using EMG recording of both the right and left homologues of the sternocleidomastoid and trapezius muscles would answer this question more clearly. A remaining age effect of head tracking with a smaller diameter would support the argument for an inability to integrate the two-dimensional motion. However, a diminished age effect would support the argument for a problem in integrating different muscles. Additional explanations could be that horizontal motion is ecologically much more common than vertical motions (Rakison, 2007), or that there is a biological predisposition to negotiate horizontal motions due to our (typically) erect position.

The age difference in timing between head movements and object motion in the vertical dimension is in line with the findings of Gredebäck et al. (2002) with regard to infants' eye tracking. In the studies by Gredebäck et al. (2002) and Gredebäck et al. (2005), the analyses of vertical and horizontal gaze points revealed a developmental difference between horizontal and vertical eye tracking in terms of vertical eye tracking being more immature in young infants. Additionally, 5-9 months old infants' 
horizontal eye tracking has been found to have a higher proportion of smooth pursuit compared to vertical tracking (Grönqvist, Gredebäck, \& von Hofsten, 2006). Note that the greater displacements seen in the horizontal dimension for both infant groups (Figure $3 \mathrm{a}$ and $\mathrm{b}$ ) are not implying a lack of control in the execution of horizontal head movements. As argued by Grönqvist and colleagues (2006), the high proportions of head movements, often seen in young infants' horizontal visual tracking (Jonsson \& von Hofsten, 2003; von Hofsten et al., 1998; 2000), are to be interpreted as used by the infants to expand the visual field.

In the present study, both younger and older infants displayed a similar developmental pattern in that vertical head tracking was more immature than horizontal head tracking. Dubrovsky and Cullen (2002) argue that information about the motion of a visual and moving stimuli drive initiation and maintenance of both eye and head. The present study, showing that head tracking displays the same developmental pattern as eye tracking, further support the argument for a common drive mechanisms involved in both eye- and head tracking as proposed by Dubrovsky and Cullen (2002).

\section{BIBLIOGRAPHY}

Daniel, B. M., \& Lee, D. (1990). Development of looking with head and eyes. Journal of Experimental Child Psychology, 50, 200-216. [Abstract/Full Text]

Dubrovsky, A. S., \& Cullen, K. E. (2002). Gaze-, eye, and head-movement dynamics during closedand open-loop gaze pursuit. Journal of Neurophysiology, 87, 859-875. [Abstract/Full Text]

Gredebäck, G., von Hofsten, C., \& Boudreau, P. (2002). Infants' visual tracking of continuous circular motion under conditions of occlusion and non-occlusion. Infant Behavior \& Development, 25, 161-182. [Abstract/Full Text]

Gredebäck, G., von Hofsten, C., Karlsson, J., \& Aus, K. (2005). The development of two-dimensional tracking: A longitudinal study of circular pursuit. Experimental Brain Research, 163, 204-213. [Abstract/Full Text]

Grönqvist, H., Gredebäck, G., \& von Hofsten, C. (2006). Developmental asymmetries between horizontal and vertical Tracking. Vision Research, 46, 1754-1761. [Abstract/Full Text]

Johnson, S.P., Amso, D., \& Slemmer, J.A. (2003). Development of object concepts in infancy: evidence for early learning in an eye tracking paradigm. Proceedings of the National Academy of Sciences, 100, 10568-10573. . [Abstract/Full Text]

Jonsson, B., \& von Hofsten, C. (2003). Infants' ability to track and reach for temporarily occluded objects. Developmental Science, 6, 86-99. [Abstract/Full Text]

Lanman, J., Bizzi E., \& Allum J. (1978). The coordination of eye and head movements during smooth pursuit. Brain Research, 153, 39-53. [Abstract/Full Text]

Price, M. C., \& Gilden, L. G. (2000). Representations of motion and direction. Journal of Experimental Psychology: Human Perception and Performance, 1, 18-30. [Abstract/Full Text] 
Rakison, D. H. (2007). Fast tracking: Infants learn rapidly about object trajectories. Trends in Cognitive Sciences, 11, 140-142. [Abstract/Full Text]

Rosander, R., \& von Hofsten, C. (2004). Infants' emerging ability to represent object motion. Cognition, 91, 1-22. [Abstract/Full Text]

Salman, M.S., Sharpe J.A., Lillakas, L., Dennis, M., \& Steinbach M.J. (2006). Smooth pursuit eye movements in children. Experimental Brain Research, 169, 139-43. [Abstract/Full Text]

Shea, S. L., \& Aslin, R. N. (1990). Oculomotor responses to step-ramp targets by young human infants. Vision Research, 30, 1077-1092. [Abstract/Full Text]

von Hofsten, C. (1994). Planning and perceiving what is going to happen next. In M. M. Haith, J. B. Benson, R. J. Roberts, \& B. F. Pennington (Eds.), The development of future oriented processes (pp. 63-86). Chicago: The University of Chicago Press.

von Hofsten, C., Feng, Q., \& Spelke, E. S. (2000). Object representation and predictive action in infancy. Developmental Science, 3, 193-205. [Abstract/Full Text]

von Hofsten, C., Kochukhova, O., \& Rosander, K. (2007). Predictive tracking over occlusions by 4month-old infants. Developmental Science, 10, 625-640. . [Abstract/Full Text]

von Hofsten, C., \& Rosander, K. (1996). Development of gaze control and predictive tracking in young infants. Vision Research, 36, 81-96. [Abstract/Full Text]

von Hofsten, C., \& Rosander, K. (1997). Development of smooth pursuit tracking in young infants. Vision Research, 37, 1799-1810. . [Abstract/Full Text]

von Hofsten, C., Vishton, P., Spelke, E. S., Feng, Q., \& Rosander, K. (1998). Predictive action in infancy: Tracking and reaching for moving objects. Cognition, 67, 255-285. [Abstract/Full Text]

\section{ABSTRACTS}

In this study, kinematic and video recordings were made of the eye- and head tracking of an object revolving in a vertical plane in two groups of infants (6- and 12-month-old) and one group of adults. The study had two purposes. The first concerned infants' abilities to negotiate the target trajectory when being forced to extensively recruit the head. The second addressed the question whether the same underlying neural controller drives both eye- and head motor systems. It was found that head tracking ability is functional already in 6-month-old infants. However, infants of both age groups displayed more extensive head movements and less accuracy compared to adults. This finding is in line with previous research on one-dimensional horizontal head tracking in infants. Infants also showed less developed timing between head movements and vertical object motion, supporting the argument that both eye- and head tracking have a common developmental trajectory.

\section{INDEX}

Mots-clés: agitation circulaire d'objet, Enfants humains, mouvement de tête, mouvement des yeux

Keywords: circular object motion, eye tracking, head tracking, human infants 


\section{AUTHORS}

\section{BERT JONSSON}

Department of Psychology, Umeå University, SE-901 87 Umeå, Sweden

bert.jonsson@psy.umu.sewww.psy.umu.se

\section{LOUISE RÖNNQVIST}

Department of Psychology, Umeå University, SE-901 87 Umeå, Sweden

\section{ERIK DOMELLÖF}

Department of Psychology, Umeå University, SE-901 87 Umeå, Sweden - Kolbäcken Child Rehabilitation Centre, Kandidatv. 31-33, SE-907 33 Umeå, Sweden 